\title{
Single image reconstruction of human heart surface with specular reflection remover
}

\begin{abstract}
$3 \mathrm{D}$ reconstruction with specular reflection remover is one of the vital and robust tools that provide aid in many fields, especially medical filed. This article presents a novel method for reconstruction a real human heart surface from a single view image with a remover specular reflection while keeping the image structure. Reconstruct a heart model from numbers of real images is difficult task and time consuming especially involve reflections, resulted from moisten of the human heart surface. In this paper, we propose a novel method for reconstruct a human heart from a single image while detecting and correcting the specular reflection. The process start with acquired the real heart image by a digital camera in cardiac surgery. Second, processed the image to extract the $\mathrm{x}, \mathrm{y}$, and $\mathrm{z}$ axes for each pixel and automatic detect the specularities using the difference of the maximum blue color channel and standard deviation of the RGB color channels. Later proceeded with the correction process by the Lshape inverse (ũ) to recover losing information saturated by lights in the operation theater. Finally, the reconstructed of the 3D model for the heart. Experimental results on the heart images show the efficiency of the proposed method comparing to the existing methods.
\end{abstract}

Keyword: Human heart surface; L-shape inverse; Specular reflection; Surface reconstruction; Image base reconstruction 